\title{
AC 2009-1711: ARE SIMULATION TOOLS DEVELOPED AND USED BY EXPERTS APPROPRIATE EXPERIMENTATION TOOLS FOR EDUCATIONAL CONTEXTS?
}

Alejandra Magana , Purdue University

Sean Brophy, Purdue University

George Bodner, Purdue University 


\title{
Are Simulation Tools Developed and Used by Experts Appropriate Experimentation Tools for Educational Contexts?
}

\begin{abstract}
Simulations and visualizations can lead to significant improvements in students' conceptual understanding. This increased understanding may be due to the formation of expertlike dynamic mental models. Laboratory simulations have been used in educational contexts for inquiry learning by allowing learners to perform experiments as they would in a laboratory. The goal of this research study is to describe the way in which an instructor and his students perceive and experience computational tools in the context of a semiconductor devices graduate course. To accomplish this, a case study research design is presented. The case study consists of two levels of qualitative and quantitative data collection. An instructor and 19 graduate students in his course participated in this study. The initial study uses a student survey administered in the fall semester of 2008. The results of these survey help to identify instructors utilizing effective instructional methods by the students. The second part of the study consists of individual case studies of the instructor and one of his students followed by a cross-case pattern analysis. Results suggest that the instructor leveraged the potential of using computational simulation tools as laboratory simulations by creating meaningful learning experiences for his students. These experiences in turn, seemed to be well perceived and experienced by his graduate students.
\end{abstract}

\section{Introduction}

The Network for Computational Nanotechnology $(\mathrm{NCN})$ is transforming nanoscience to nanotechnology by providing researchers high-end, research quality, online simulations to 89,000 users for the year 2008 (Lundstrom et al., 2008) ${ }^{1}$. A web portal called nanoHUB.org was initially focused on pioneering the development of nanotechnology from science to manufacturing through innovative theory, exploratory simulation, and novel cyber-infrastructure. Many participants in the center have incorporated the nanoHUB.org simulation tools as well as other resources to support the education and training of graduate and undergraduate students new to specific areas of nanoscience. Therefore, our main goal is to investigate how these expert tools can be used as learning tools.

The nanoHUB.org provides research-quality simulations that experts in nanoscience commonly use to build knowledge in their field. NanoHUB.org leverages an advanced cyberinfrastructure and middleware tools to provide seamless access to these simulations. As described on the nanoHUB.org website, key characteristics of the nanoHUB.org simulation tools that make them good resources for incorporation into classroom environments are: a) they were produced by researchers in the NCN focus areas, b) they are easily accessed online from a web browser powered by a highly sophisticated architecture that taps into national grid resources, and c) they provide a consistent interactive graphical user interface-i.e. Rappture, which makes even the most esoteric computational model approachable to experts and non-experts. Rappture is a toolkit that allows the incorporation of a friendly graphical user interface with the simulation tools in the nanoHUB.org (McLennan, 2005) ${ }^{2}$. For example, Figure 1 illustrates a typical 
user's interface overlaying a highly sophisticated computational model of a nano-transistor model.

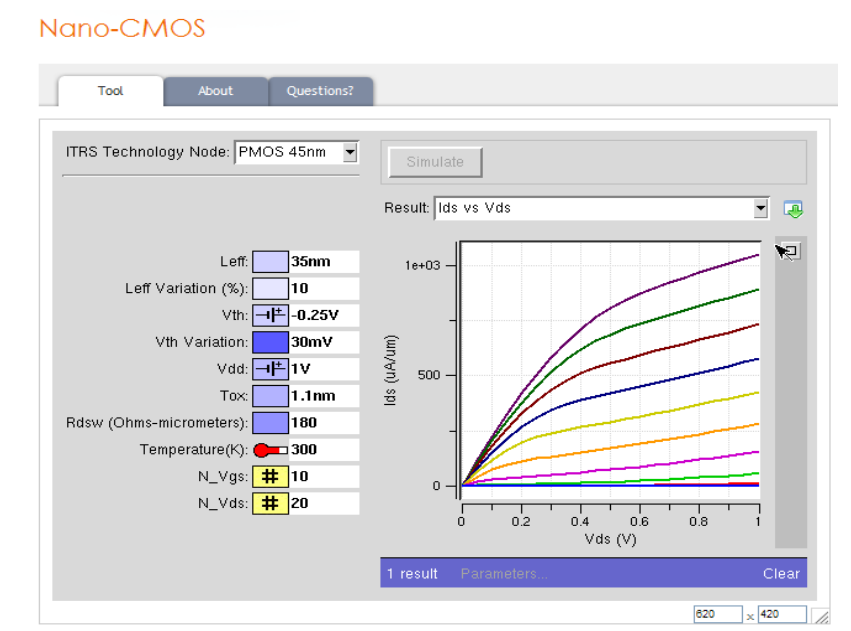

Figure 1: Nano-CMOS interface of nanoHUB.org simulation tools

Our initial step is focused on investigating how students, from a course in semiconductor devices perceive simulations as learning tools. Our guiding questions include: Can simulation tools developed and used by experts also be used as an educational resource for apprenticing new talent into nano-engineering? What are graduate students perceptions of the utility of these resources for learning?

\section{Review of the Literature}

Cannon-Bowers and Bowers $(2007)^{3}$ defined simulations as "a working representation of reality; used in training to represent devices and processes and may be low or high in terms of physical or functional fidelity (p. 318)." In an educational context, Alessi (2002) ${ }^{4}$ proposed that simulations are "any program which incorporates an interactive model (one which can be repeatedly changed and rerun) and where the learning objective is for students to understand that model, whether through discovery, experimentation, demonstration, or other methods (p. 177)." For our purposes, we define simulations as -

A simulation is an interactive computational model with user control of specific variables (inputs) and multiple methods for displaying common relationships of interests (outputs, e.g. graphs) to experts (scientist perfecting the models or engineers using them to design devices).

Simulations, or interactive models, are tools of inquiry used by experts to achieve their goals of explanation and prediction of a systems' behavior. Alessi (2002) ${ }^{4}$ and de Jong and van Joolingen (1998) ${ }^{5}$ identified "model-exploration simulations" for developing and understanding of the behavior of a phenomena and models capturing such behavior. Simulations contain models representing specific concepts, facts, and principles within a domain. In an educational setting, the ultimate purpose of this type of simulation is to allow learners to explore a models' behavior (Clariana \& Strobel, 2007) ${ }^{6}$. Model-exploration is conducted when learners test an 
input-output relationship (Du Boulay, O'Shea, \& Monk, 1999) ${ }^{7}$. Model-exploration simulations have the following characteristics: a) the software simulations are purpose/domain specific (Cannon-Bowers \& Bowers, $2007^{3}$; Clariana \& Strobel, $2007^{6}$ ), b) the software simulation does not allow the learner to alter the system's underlying model (Cannon-Bowers \& Bowers, $2007^{3}$; Clariana \& Strobel, $2007^{6}$ ) unless it has predetermined options, c) the software simulations request input parameters from the learner (Cannon-Bowers \& Bowers, 2007) ${ }^{3}$, and d) the simulation tool displays some sort of output (Cannon-Bowers \& Bowers, $2007^{3}$; Clariana \& Strobel, $2007^{6}$ ). These types of simulations include a black-box model in which the calculations are hidden to the user and the relationships between variables must be inferred (Alessi, $2000^{8}$; Resnick, Berg, \& Eisenberg, $2000^{9}$ ). Therefore, the models and type of inquiry to investigate it is much like the inquiry used in the natural sciences. The underlying assumption is the system is consistent and repeatable.

According to Alessi $(2002)^{4}$, laboratory simulations are also a type of model-exploration simulations and have the purpose of allowing learners to perform experiments as they would in a laboratory; for example, by using a physical device such as a measurement tool. This particular type of simulation also includes a black-box model. However, in this case the main purpose of the laboratory learning experience is not focused on inferring the relationships between variables, but on the skills and procedures for conducting an experiment, such as doing a titration, determining an unknown substance, characterizing a device and so on.

Simulations, in educational contexts have been used for inquiry learning. According to Pizzini et al. (as cited in Roth, $1993^{10}$ ), inquiry learning involves activities such as:

(a) the identification of problems and solutions and the testing of these solutions;

(b) the design and students' own procedures and data analyses; (c) the formulation of new questions based on previous claims and solutions; (d) the development of questions based on prior knowledge; (e) the linking of experience to activities, science concepts, and science principles; and (f) the sharing and discussing of procedures, products, and solutions. Problem solving is an inherent feature of open inquiry and an important issue in the training of scientists and engineers. (p.166)

Studies, such as those conducted by Williamson and Abraham (1995) ${ }^{11}$, have shown that the use of computer-interactive animation technology and dynamic, three-dimensional presentations can lead to significant improvements in students' understanding of the concept in question. They argued that this increased understanding may be due to the superiority of the formation of more expert-like dynamic mental models. However, researchers have agreed that studies related to the use of computer simulations for inquiry learning have not demonstrated compelling evidence of their effectiveness in science and engineering domains (Njoo \& de Jong, $1993^{12}$; Winn, $2002^{13}$ ). Njoo and de Jong (1993) ${ }^{12}$ pointed out two difficulties encountered when incorporating simulations into educational contexts. The first is that the exploratory learning processes may be too difficult for learners, and/or that students may not use their exploratory skills even though they possess them. In addition, Bodemer et al. (2005) ${ }^{14}$ suggested that learners may lack pre-requisite knowledge. Another difficulty, perhaps, in the case of 
building simulations, results from the complexity of the modeling task (Clariana and Strobel, 2007) ${ }^{6}$. However, most of these studies have employed quantitative research methods and therefore may not have taken into account the interaction of different variables that usually take place in complex learning environments. Such may be the case of the influence of students' expectations of the task or the effect of direct instruction. Consequently from a research design point of view these studies lack ecological validity; from a practical point of view, there is a lack of practical application of previous findings (Winn 2002) ${ }^{13}$.

The goal of this study is to document how an instructor and his students perceive and use simulation tools in their learning environment. Our guiding research question is:

What are instructors and students' perceptions and experiences of nanoHUB.org simulations as learning tools in the context of a graduate engineering course?

Over the past four semesters approximately 360 undergraduate students and 190 graduate students participated in our ongoing study involving nanoHUB.org learning resources in higher education learning environments. These students represent a population of 16 different engineering courses at eight different universities. This initial study concentrates on analyzing one graduate level engineering course in semiconductor devices to describe one instructional model for using simulations for learning and describing in detail a student's perception of this method.

\section{Methods}

The goal of this research study is to describe an instructor's and his students' perceptions and experiences with simulation tools as laboratory simulations in the context of an advanced graduate elective course for electrical engineering students. These graduate students are specializing in the area of very-large-scale integration (VLSI) and circuit design. The course focuses on examining advanced transistors and its physical principles. Considerations that enter into the development of new integrated circuit technologies were also explored. This course has as pre-requisite a course related to solid state devices only offered to master's student standing or higher. This pre-requisite course involves a relatively-broad, moderate-depth coverage of semiconductor devices and related topics.

An instructor and 19 advanced graduate students in electrical engineering participated in this study ( 2 females and 17 males). Interviews were conducted with the instructor and one of his students. The interview with the instructor was done before the semester started and the interview with his student was done after the first homework assignment. The survey data was collected at the end of the semester. The follow outlines both instruments and the analysis methods used to interpret the results.

\section{$\underline{\text { Survey Instrument }}$}

The study begins with a quantitative study using an anonymous student survey given to graduate students participating in a course on semiconductor design at the nanoscale level. The 
survey was administered in the fall semester of 2008 to 47 students and 19 students completed the survey. The survey was designed with two main intentions: a) monitoring the usage of the nanoHUB.org and b) assessing instructors' incorporation of the tool(s) as part of their course. The students were asked to participate in a voluntary Likert-scale survey focused on:

- How students perceive simulation tools as useful for their learning, - How students thought the simulation tools were relevant to their areas of interest and their level of satisfaction, and

- Usability aspects - in particular, how intuitive the tools are.

Students responded using a scale from one to four: strongly agree, agree, disagree, and strongly disagree to each question. The students responded anonymously using an online version of the survey at the end of the semester. The response rate for the survey was approximately $40 \%$ of the entire population of students in this course.

\section{$\underline{\text { Interview Protocols }}$}

We selected case study research design as the most appropriate qualitative research design to capture the complexity of a single activity and its important circumstances (Patton, $2002)^{15}$. The second part of the study consisted of focused interviews with the instructor and one of his students. The instructor's interview protocol captures the instructors' intentions for learning outcomes, assessment and pedagogical approach for teaching with the nanoHUB resources. The interview with the instructor was done before the semester started.

The student interview protocol aligns with the goals for the survey and focuses primarily on students' response to the benefits of the tools for meeting the course objectives and their overall learning goals for the course. However, the interview with the student was conducted in the context of a particular homework assignment. The case study consists of results for each of these data sets and a cross-case pattern analysis between instructor's and students perceptions of learning outcomes, assessment and pedagogical methods. This analysis is described in more detail in the results section. The interview with one of the instructor's students was done after the first homework assignment and before feedback was provided to her.

\section{Analysis and Results}

The quantitative analysis of the student survey data served two main purposes: (1) to provide an initial indicator of students' perceptions of the professors' incorporation of simulation tools in the learning experiences and (2) to focus on the design of our interview protocol with professors and students.

The qualitative analysis of the interview protocol data provided additional details of instructors and students perceptions and experiences. This level of detail furnishes additional details that can be aligned with the information from the student survey. Our goal is to use this data to inform and improve instructional methods using nanoHUB.org resources. The student survey Likert scale items were translated into numerical values (Strongly Disagree $=1$ to 
Strongly agree $=4$ ). The average rating and standard error (error bars) are summarized in Figure 2. The four point scale was designed to encourage students either agree or disagree with a statement. Therefore, we assume that items with the standard error one standard deviation away from the neutral range (2.5) are an indication of a main effect.

The results of the survey and interview protocol were organized around Wiggins and McTighe's backward design (1997) framework for designing effective learning experiences. Wiggins and McTighe presented a "backward design process" (p.9) composed of three main stages: a) identifying the desired learning outcomes -- the content of the lesson, b) determining the acceptable evidence of learning also called the assessment method, and c) planning the experiences and instructional approach or pedagogy. We added Usability aspects to evaluate quality of students experience using the simulation since this could have an affect on their response. The results from this initial study are summarized in Figure 2 and elaborated below.

Students' survey responses

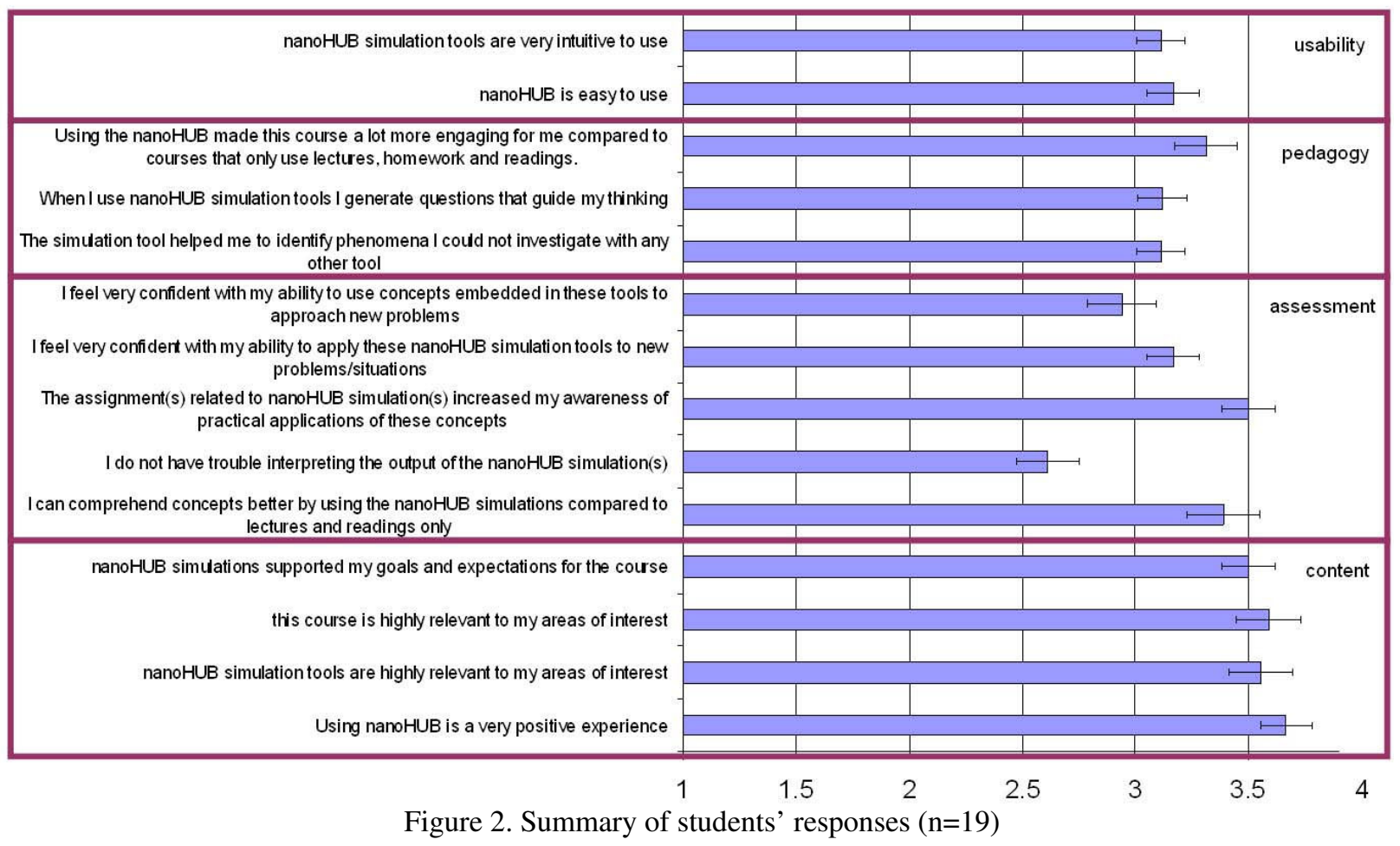

Learning Outcomes- This category of items focuses on the general experience students had, whether students thought the simulation tools were relevant to their areas of interest, as well as their level of satisfaction. Graduate students considered the nanoHUB.org as a positive experience as well as being relevant to their areas of interest. Students had also responded positively to the idea that the nanoHUB.org simulations supported their goals and expectations for the course. Students also considered the courses whose instructors incorporated the nanoHUB.org simulations as highly relevant to their areas of interest.

Evidence of Learning- This section focuses on how students perceive simulation tools as useful for their learning and how they may transfer it to practical situations. Students were 
positive in perceiving that using the nanoHUB.org simulations allowed them to comprehend the concepts better in comparison to students who only have access to lectures and readings. There was also a positive tendency in the responses of students' awareness ability to guide their own inquiry by generating and answering their own questions.

Students were moderately positive in their ability to interpret the graphical outputs of the simulations. Students were also moderate to positive in their ability to use the concepts embedded in the simulation tools to approach new problems. We are investigating this as part of our ongoing research.

Instructional Approach -This section identifies whether the simulation tools were learning tools. Students reported positive responses to using nanoHUB.org simulation tools to generate questions that guided their thinking, and also positively reported that using the nanoHUB.org made the course a lot more engaging for them compared to courses that only use lectures, homework, and readings. We also asked students if the simulation tools helped them identify phenomena they could not investigate with any other tool. Students considered the simulations tools helped them identify phenomena they could not investigate with any other tool.

Usability -Students reported that nanoHUB.org simulations are very intuitive as well as easy to use.

\section{Case Study 1: Dr. Sanders}

Dr. Sanders is a faculty member in the electrical engineering program at a large Midwestern university. Dr. Sanders has more than 30 years of engineering teaching and research experience. Before joining academia, Dr. Sanders gained corporate experience working for the integrated circuit industry. Dr. Sanders's teaching and research have been recognized with several awards.

The course Dr. Sanders taught was a graduate elective course for students in electrical engineering specializing in the area of VLSI and circuit design. The course focused on examining advanced transistors and its physical principles. Considerations that enter into the development of new integrated circuit technologies were also explored. These considerations ranged among process, device, circuit, and systems perspectives. The content of the course was divided in three main topics: MOSFET fundamentals, short channel MOSFETs and CMOS processes, and CMOS circuits plus new materials and structures. The structure of the class consisted of lectures that met two times per week for a period of 75 minutes and weekly homework assignments. Online lectures were incorporated too, sometimes instead of a lecture and sometimes as part of homework assignments. These online lectures were accessible to students through the nanoHUB.org. Most of the online lectures Dr. Sanders assigned were recorded by him; however, he also incorporated lectures from other researchers who have given open access to them through the nanoHUB.org. The assessment criteria for the course focused on three exams, and the homework assignments; each of them with a value of $25 \%$. The simulation tools were incorporated as part of the homework assignments. 
Dr. Sanders Experiences of the nanoHUB.org Simulation Tools for Instruction

\section{Learning Outcomes}

Dr. Sanders has been using several of the nanoHUB.org simulation tools as part of his graduate course in semiconductor devices. He used the nanoHUB.org throughout the entire semester and at different points in time. Dr. Sanders's instruction focused on helping students "developing an intuitive understanding" of the phenomenon under study. He did that by designing learning activities that either would imitate a lab experience, or activities researchers would conduct for industry development.

... this homework eight that I have here, so this was like a virtual lab. These are the kind of measurements that device engineers frequently go in a lab and do, and it gave them a chance to do it by simulation...

Throughout the nine homework assignments, Dr. Sanders followed an approach in which each homework assignment was part of a progression in the level of complexity. The last one though, he targeted to integrate all the knowledge gained through the semester which he defined as a "design challenge".

Now, you know, then maybe the most challenging one was this scaling exercise, that's homework nine, where they tried to use all of the knowledge they had in class about what controls the performance. Those are the issues when you make the device smaller...

According to Dr. Sanders, this activity not only served as a final project integrating all the knowledge of the semester, but also it tried to imitate an industry design experience.

... so this particular one, sort of integrates all of the knowledge in the course, and kind of explains, this is... if you are going to be a device-development engineer in Intel or something, this is basically what you are going to be doing, trying to do work like this, if you are a circuit designer or someone who is just using that technology to understand how the devices operate, you know, this is in what Intel spends two or three years on every time they move to the next technology generation...

\section{Evidence of Learning}

Dr. Sanders focused his assessment in students designing devices to meeting industry target parameters. While in one assignment he asked his students to "look at a paper that presents some measured data from a current generation" and asked them to "tweak the parameters in the model so they can get a best fit"; in the final assessment he went beyond making it a "design challenge," asking his students to meet parameters of a next generation device.

Since most of the activities related to the nanoHUB.org simulation tools were related to homework assignments, solutions were posted on the website. After students saw their responses and had a chance to corroborate them with the solution provided by the instructor, Dr. Sanders 
scheduled feedback sessions outside lectures. When I asked him about the process of providing feedback Dr. Sanders said:

Yeah, that's the problem, in the course I didn't have enough time to do that. We had a couple of help sessions that we scheduled in the evening and we went through a couple of homework assignments explaining... this is what you should have learned from the homework assignment... probably we didn't do as many of those as we should have... hmm so my challenge the next time.. I think, there wasn't enough of that... we posted the solutions, but I think that really needs some discussion.

Dr. Sanders identified that probably this feedback is not enough, but he mentioned his willingness to try different arrangements taking advantage of the fact that he recorded all his lectures the last time he taught the class. These lectures are uploaded on the nanoHUB.org and have an easy access for students.

We had maybe two or three, possibly four but it was not enough. I was even thinking that I can use some of the recorded lectures and instead of using the class. Because with all the material I had to cover, it was very difficult to devote class period to discuss the homework, so I may try next time to assign, ok listen to the lecture on your own time and now I'm going to use class time to discuss homework assignment this time. I don't know how that's going to work.

\section{Instructional Approach}

At the macro level and with the exception of homework one, the most common instructional approach followed by Dr. Sanders was to first introduce the concepts during class and then assign the homework. Homework assignments were designed by Dr. Sanders with the intention to provide practice for students. At the micro level most of the times the homework assignments were focused on exercises that allowed the students to compare those concepts learned in class, with results from the simulations. For example, for the case of homework two:

They took the analytical calculation they have done in class and they did that first, and then they ran a simulation tool and they compared results and explain what was similar and what was different...compared theory versus simulation. They do some of the quantities that we were talking about in class and compared them to with what the theory says is should be.

Dr. Sanders explained that while the exercises done in class are approximation to the exact solution, with the simulation tool students can solve the exact ones. Therefore students had the opportunity to compare those and draw some conclusions and identify "how it really works".

...we have to make lot of simplified approximations so it work out, you know equations for this, hum... that you really go about it in practice, now we are going to run a simulation that solve the equations properly without these approximations, and we are going to see how it really works and which of these approximations are good and which of them aren't so good and things like that. 
For the case of the first homework assignment Dr. Sanders designed it as an exploratory activity. Dr. Sanders gave an opportunity for the students to get familiarized with the simulation tool as well as with the output and what that output means.

So if I look at this first one it's just that, this one most students don't have any hands on experience with transistors, they don't know what the current/voltage characteristics look like, so we just want them basically get them some experience this device has three terminals, you hook it up and apply voltages, these are the currents that you measure, this is how a typical device behaves, this is how much voltage you typically apply, you know 1 or 1 point 2 volts, this is how much current typically get. So it was just to give them before they start the course to get them some feel for how these devices work, what voltages you apply to them and what currents, flow...

As part of Dr. Sanders's pedagogical approach, he usually provided to his students a starting point. Sometimes it consisted on a model to be tested in the simulation tool, some initial parameters that should be met, and/or some others consisted of a model to be implemented (i.e. writing a Matlab script) outside the nanoHUB.org. Then, the output of such model became the input for the nanoHUB.org simulation tools. For example:

Well so I guess, you know, what we give the students as a starting point is a current generation device that behaves well, and we tell them ok we want you to shrink the size of this device and has to perform in this way, so the first step would be just to go and properly reduce the dimensions...

\section{Dr. Sanders Perceptions of the nanoHUB.org Simulation Tools}

Dr. Sanders considered nanoHUB.org simulation tools as convenient because they allowed his students to solve hard analytical calculations easily through simulation. Another feature of the simulation tools mentioned by Dr. Sanders is that his students had the capability to use different models run with different parameters that would run different outputs.

...so I can choose where I want the plot... let's turn that off just to save time, but normally they would want to plot that too, so this tells them what kind of output plots they would get through the terminal characteristics of the device that like you are measuring if you want to do it on a lab...

Dr. Sanders made clear that nanoHUB.org simulation tools have a high level of complexity that is transparent to the students; as it maintains a simple user interface.

Yeah, now this one is actually... it has a simple interface underneath it, is an industrial strength tool underneath,... it's one that was developed at Bell Labs a few years ago, so the simulation that's doing is nontrivial, it might take a few minutes here... Now what we've tried to do is to simplify the interface because it would typically take a graduate student maybe a few weeks to learn the full interface to run in this tool. This is the kind of tool that you would run in industry hum... it can do a lot but there's a complicated 
input format that you specify in the file and we really don't want to use class time for all of that, you can easily spend a week just explaining how you run the tool, how you set up an input file and we are trying to eliminate all of that.

Dr. Sanders also mentioned that a specific element of simulation tools which make them unique was that it gave him an additional capability that could not be replicated on the lab and visualize the data.

So now, if I want to go in, I can look inside the device, so that's the kind of data I would just give them in the lab and measure the device, but what the simulation allows you to do is to look inside the device and if I want, I can take a look and see a electric field inside the device... let's see if we can...

\section{Case Study 2: Valerie}

Valerie is an electrical engineer graduate student who attended Prof. Sanders' lectures for a semester long course. This is the first time Valerie used nanoHUB.org simulations; however she has experience using other computational tools. Although Valerie's main research is in the area of microwaves, she is very interested in the area of semiconductor devices and has had lot of graduate course experience on that topic. The following descriptions represent Valerie's perceptions and experiences of simulation tools in the context of homework assignment one.

According to the homework assignment's description, the task consisted of examining the IV characteristics of $45 \mathrm{~nm} \mathrm{~N}$-channel CMOS technology. The professor then requested for specific parameters (e.g. on-current, off-current, sub-threshold swing, etc.) and a description of how they obtained each parameter. Professor Sanders also pointed out the possibility to change the minimum and maximum axes scales and to select either linear or logarithmic scales. The second exercise consisted of repeating the same problem for a p-channel MOSFET by selecting PMOS $45 \mathrm{~nm}$ and specify the same parameters. The last exercise consisted in using the simulation tool in a "discovery" mode to determine which technology parameters (e.g. Leff, Vth, Vdd, Tox, Rdsw, temperature) have the strongest effect on DIBL.

Valerie's Experiences of nanoHUB.org Simulation Tools for Learning

\section{Learning Outcomes}

Valerie believed that this topic and strategies to learn it require a lot of conceptual understanding of the physical phenomena.

...solid state I believe is one subject where you have to sit down and understand the physical phenomena and its not like electromagnetics is more math and um, this I believe is more of understanding the physics,

Valerie identified that the main idea of the particular topic of the homework was to identify that as the size of the device gets smaller, the physics change. In addition Valerie also identified that Dr. Sanders' goal was for them to get a feel of the typical values of the devices at 
different scales and which of those are changing the most.

... from the lectures he was trying to insist that as we decrease the size of the device, things would be a lot different. So I think uh, the reason why he wanted us to use this was because uh, first thing to get an idea about how, what are the typical values like for resistance, for the channel resistance, for the change resistance, for the on current, for the off current, all these things, what would they be, what would be the typical values of such parameters at 45 nanometers, that is the first idea. And then, ... because this course is all about small device transistors, nano scale transistors, so um, it, this tool would be very useful even to compare with a larger transistor and see how it would, how it would change, so I think that was his idea for us. Um, because we never compared stuff with larger technology devices, I think the main reason he wanted us to do this was to get a feel for all the parameters at the 45 and 100 slope, How does the 45 nanometers at most behave, how does the DIBL behave, and that kind of stuff.

Valerie mentioned that she feels pretty confident with this particular topic of the course. She explained that she has taken similar courses in the past but nothing specific to small scale devices. However, by attending the lectures and the additional materials that the professor provided them on the nanoHUB.org was enough to complete the assignment successfully. She also provided us with some evidence on her prior knowledge and confidence about the topic:

I have taken solid state courses all through my masters and um, after coming here, I couldn't take any solid state courses and this is my first one here, so but when I gave my qualifying exam, I did three questions in solid state and one question in a different topic, and pretty much, I got it on points in the solid state, so that's why I like solid state, studying it and all it seems, it makes sense to me even though it doesn't make sense to some people. Like for me, solid state is one subject which makes real sense to me.

Evidence of Learning

After completing the assignment Valerie gained better understanding of prior knowledge. When we asked her what has she learned form the activity she said:

... I know I have studied MOSFET, but I don't remember was exactly is on current, what exactly is DIBL, I think now I know kind of, what is on current and what is off current, I didn't know there would be two separate like threshold voltages like VT(lin) and VT(sat), which um, Professor explained in the lecture. Yeah, the online presentation which was part of the homework too, first go through the lecture and then do the homework. So um, like I didn't know there would be two threshold voltages and which one was what. Um, I didn't have clear understanding of MOSFET, I was familiar with the MOSFET, but um I didn't have like a clear understanding of MOSFET. Um, and uh, and its different when you study something in detail like this and its different when you are browsing through, learning MOSFET as a new device, you wouldn't be concentrating observing so much where is on current where is off current, you maybe learn how the device operates and you know that kind of stuff, I have never learned MOSFET quite in detail. 
Specifically from the simulation tool, Valerie learned how to get and read the parameters from the tool:

...first we just plotted these curves and then we plotted a log ID versus VDS curve and most of the parameters can be got from log ID versus VDS curve, you can get VDSAT, threshold swing, what is on current, off current, so that is entirely, like finding out the parameters from one curve that was very different for me, like I have never learned that. Now I know...

Valerie also gained new knowledge, like how to check different parameters and new ways to characterize devices. Valerie was able to identify new situations where to apply what she has learned so far from the course and she posed the example of an internship she did in the past:

I think uh, I think like um, this time for my internship I um, I was looking on a different device, like not exactly a different device, it was a same device but um a scaled down version of the same device, and I was asked to characterize that device. Like um, that was one of the main tasks that I was doing in the internship, so um, so that time I used a different tool, now if I would like I could always use this to do it, and moreover now I know different ways of checking whatever the different parameters, so through this homework through the course and everything, so it will be very helpful, I could always use it if I need it to characterize any device in the future because I was general IV curves when I was characterizing the device, now I know that I can also characterize the device using um, Log IV versus VGS curves too, and then see what's happening.

However, Valerie found one portion of the homework assignment a little bit challenging. In particular she thought the difficult part was to calculate or evaluate which parameter Changes the DIBL of the device the most. Part of the difficulty she had was because she was struggling with the interface. Valerie ended up extracting the data from the simulation tool and actually plotting it in Excel. She explained us how she struggled:

...the whole assignment for me, the toughest part was the third question where we had to calculate, or evaluate which parameter changes the DIBL of the device. So, it was very difficult for me to, you know I could change the parameters, I could have all the results on the same plot, but unless I grab data from each of the files, and then calculate DIBL in each individual case, it was very different. Lets say for instance I change um, I change the device width, uh, sorry the device the length, the effective length of the device, and then I notice that this is the change, so um, I have ok, if I have this much of the device length, this much of a change of that, then this is the DIBL that I am getting. So I observe the trends of each individual thing, let's say I change just the effective length, I know all the trends. I change it from 20 nanometers to 60 nanometers and then I saw, and then I did the same thing for changing each parameter, and then I was able to see the results. Then, I did this trick where you know, lets say my DIBL is decreasing as I decrease my effective length, so I took my lowest value of the effective length and then the rest of the parameters as a same thing, and then I plotted a curve and then I took the highest value of thickness of the oxide and then I put it in, and I saw the value and then compared those 
but it was very difficult for me to actually see, which DIBL unless I, you know take the data for each individual case, plot it in Excel or something and calculate the DIBL in each case and see which one is giving me more effect.

\section{Instructional Approach}

Valerie identified some of the instructional approaches of Professor Sanders as very useful. First of all during lecture time, Valerie mentioned Prof. Sanders provided her with the required prior knowledge to complete the assignment. Then, the online lecture he uploaded to the nanoHUB.org was an excellent scaffold for her to complete the homework. Actually, she was reviewing the online lecture over and over and solving the homework step by step guided by the video. When we asked her if her prior knowledge was enough she responded:

Um, I kept referring back to Professor's notes, like his lecture. I was like, I listened to the whole lecture once, and then um, like lets say I had to do on current, I went back and was looking at what is on current, and then uh, I kind of knew the whole thing but it was just too much stuff to remember, so I had to you know look at Professors lecture and pause it, and then do this, that kind of stuff.

She also appreciated the benefits in her learning when the professor asked them to give their explanation of the answer; however, she expects the same from the professor when he gives the feedback to them:

... on my question three I don't know if that would be wrong, but I would definitely like to see simulation results showing you know, this is the correct answer, if you do this then this is happening, or do this then this is happening, kind of rather than just telling me, you know these are the two factors which affect the DIBL, which affect the DIBL most, so that wouldn't tell me anything, So if he could give me like plots saying like see if you do this then this is what's happening, because we were asked to do that in the homework. It's not like I could just Google it and say ok these two affect the most and then I could just write that. That is not how we do homework right, we need to explain what we are doing and need to really understand, so it really, if I really want to learn, if the professor wants me to learn and everything, he should give me the proof of why that is happening.

Valerie thought it would be appropriate to accompany the simulations with the corresponding formula or theory:

..., I know the physical phenomena, but ... the formula and everything, you can always, they are always there, like lets say I don't know how to calculate GN, I Google the formula and then I try to calculate it, so um, yeah I mean, whenever there is a simulation there basically result to the formula, the part of the theory.

The following question was if she thinks Dr. Sanders will eventually provide that information during lecture time. Valerie replied:

I think he covers it, but ... he does it pretty fast. Like its never... there is no formula 
which is stated in the lecture without an explanation for sure, he always explains it and everything, but ..., he doesn't emphasize it, he doesn't say ok this is what's happening, ... but, on the exam, I hope he won't give us questions like that ...

\section{Valerie's perceptions of nanoHUB.org simulation tools}

Valerie found nanoHUB.org simulations very useful and very simple to use. She also pointed out the easiness with which she can have access to this technology and the free access. However, she pointed out it was a little bit challenging to do specific actions and suggests making the interface more user friendly. Specifically Valerie had difficulties marking specific points on the graph with the cursor. When we asked her what was about the simulation tool that needed to be more user friendly she responded:

...with the parts where there was nothing like using the cursor, you couldn't mark like lines or anything on the graph and see what is the x position y position, all you could do is take data and then put it into something like Excel or something,

Specific capabilities that Valerie pointed out as useful in addition to easy access were the flexibility of the tool to allow the overlap of different curves and that helped her to compare the behavior of the device at different scales:

... the most important thing is that its easy, like you can log into at any technology device, like 30 nanometer or 40 nanometer, like they have a bunch of stuff and you can always compare results, so I can choose NMOS 45 nanometers and I can choose NMOS 100 nanometers and then put them on the same plot and see how they differ and everything. So uh, which otherwise it would be very difficult, but getting the curves, it uh, it was very simple, all you had to do was like click and then just get the technology that we need and then give all the parameters that were right there and then it was simple and very easy.

However, although she mentioned that it was useful the ability to overlap the curves it was difficult to distinguish all but the latest one, since only one curve is highlighted while the others have the same color and no legend was provided of what inputs corresponded to which outputs.

A second disadvantage pointed out by Valerie is related to the limitations of the tool. Valerie said that it would be very helpful to have additional curves:

I wanted to see GM versus VGS as ID curves, but there is no option to do that. The only two plots that it would let us plot um, one is the ID curves, the ID versus VDS curves and then the other one was the ID versus VGS curve and that would, yeah it would plot it in both block scale and non-block scale, it was the option to choose that, but it wouldn't let us plot GM versus ID curves, which I believe is also a very important curve which um, which lets us see where the device is struggling, where the device is weakening, where the device is in threshold and all that stuff. 
Later in the conversation she mentioned again:

The curves were very helpful, but there weren't many curves, I mean there weren't ... real good options, ... lets say I have to differentiate the ID with VGS and there was no option then for me to use a derivative, ... I can not plot ID plus something else versus something else versus something else... I didn't even have ... the stuff that you need for the MOSFET, I mean forget about transform and stuff, we don't need that for all this, but ... it didn't have ... how to calculate .. IV with derivative, or VGS...

\section{Discussion}

While Dr. Sanders pointed out three advantages of nanoHUB.org simulation tools i.e. easiness to solve complex calculations, simple interface, and additional capabilities; the most salient advantages for Valerie were the simplicity of use, easy access and flexibility of the tool to show the same data in different ways. This is consistent with students' responses to the survey that the simulation tools are easy and intuitive to use. However, Valerie pointed out that additional features could help her more in her learning experience. In specific, she wanted to be able to distinguish different curves when the simulation tool was run multiple times and suggested including additional (different) outputs of the data.

Dr. Sanders reported that his overall goal of using the simulation tools was to give his students a sense on how investigations must be conducted in their areas of expertise as well as ways in which professionals work on those disciplines. Similarly, Valerie was able to identify the same goal, which in the context of the first homework assignment was to get a feel of the typical values of the devices at different scales and which of those are changing the most. From the survey data we can identify that overall students considered that the nanoHUB.org simulations supported their goals and expectations for the course as well as the course content as highly relevant to their areas of interest.

Dr. Sanders' formative assessment method was to first have students submit the homework, then he graded it, and posted the solutions on the website. This method was the most time effective but Dr. Sanders mentioned he would like to give more refined feedback to his students. Valerie expected Dr. Sanders would provide detailed feedback. By looking at the solution Dr. Sanders uploaded to the course website, we were able to identify he gave a detailed explanation. We don't know if this explanation was enough for Valerie since we interviewed her before she received the feedback. However, by Valerie's explanation, we identified that just by doing the homework Valerie was able to have a clear understanding of the devices, reinforce prior knowledge of how devices behave at different scales, and learn about new ways to characterize devices. Work on formative feedback is continually being investigated to better understand what has the most lasting impact on students' long term understanding of the content. Direct feedback can help them understand what they did wrong, but does not encourage them to evaluate the problem in the context of future learning. One argument is that the posting of solution that requires students to compare and contrast their solution with the presented one can lead to strong learning outcomes than direct repair of specific problems (Black and Wiliam, 1998 $)^{16}$. The students who responded the survey also experienced the simulation tools as useful 
for their learning in comprehending the concepts better and in their ability to use the concepts embedded in the simulation tools to approach new problems.

The final stage in the Backward Design Process (Wiggins and McTighe, p.9) ${ }^{17}$ is the planning of learning experiences and instruction. When instruction was accompanied by a complex simulation tool, Dr. Sanders pointed out that he did not only take the time to explain concepts related to the phenomena in study, but also spent some time explaining how to operate the simulation tool. Additionally, Dr. Sanders also mentioned he posted online lectures on the nanoHUB.org that might work as guidance for students in doing their assignments. By talking with Valerie we identified that Dr. Sanders' approach of the course was perceived as useful. Valerie mentioned Prof. Sanders provided her in class with the required prior knowledge to complete the assignment. Additionally, the online lecture corresponding to the first homework assignment was a valuable and useful resource for Valerie in order to complete her assignment. Additionally, students who answered the survey reported considering the simulation tools as engaging for their learning as well as a means to identify phenomena they could not investigate with any other tool.

\section{Conclusion}

From the analysis provided above we conclude that Dr. Sanders leveraged the potential of using nanoHUB.org simulations by creating meaningful learning experiences for his students. In particular, he has used expert simulation tools as an inquiry device toward goals of designing a device. He engaged learners by using a laboratory simulation that allowed his students to characterize devices at different scales. By using expert simulations as laboratory measurement tools Dr. Sanders have created learning experiences that helped his students identify that as the size of the device gets smaller, the physics change. With this learning experience Dr. Sanders has helped his students develop an intuitive understanding of the typical values of the devices at different scales and which of those are changing the most. In addition, the students learned to generate their own questions toward these goals and run experiments to better understand how the device might work at the smaller scale. We believe this model of instruction helps students develop transferable skills to other design tasks using the nanoHUB.org simulation tools and other computational tools. These experiences in turn, seemed to be well perceived and experienced by his graduate students. 


\section{Bibliography}

1. Lundstrom, M. S. a., Adams III, G. B. a., Klimeck, G. a., McLennan, M. a., \& Potrawski, M. (2008). Network for Computational Nanotechnology Sixth Annual Report Purdue University.

2. McLennan, M. (2005). Add Rappture to Your Software Development.

3. Cannon-Bowers, J. A., \& Bowers, C. A. (2007). Handbook of Educational Communications and Technology. In J. M. Spector, M. D. Merril, J. J. G. van Merrienboer \& M. P. Driscoll (Eds.), (pp. 317-327): Mahawa, NJ: Lawerence Erlbaum Associates.

4. Alessi, S. (2002). Building versus using simulations. In Integrated and Holistic Perspectives on Learning, Instruction and Technology (pp. 175-196): Springer Netherlands.

5. de Jong, T., \& van Joolingen, W. R. (1998). Scientific discovery learning with computer simulations of conceptual domains. Review of Educational Research, 68(2), 179--201.

6. Clariana, R., \& Strobel, J. (2007). AECT Handbook of Educational Communications and Technology. 3rd. Ed. In J. M. Spector, M. D. Merril, J. J. G. van Merrienboer \& M. P. Driscoll (Eds.), (pp. 329-344): Mahwah, NJ: Lawrence Erlbaum Associates.

7. Du Boulay, B., O'Shea, T., \& Monk, J. (1999). The black box inside the glass box: presenting computing concepts to novices. International Journal of Human-Computer Studies, 51(2), 265--277.

8. Alsessi, S. (2000). Designing Educational Support in System-Dynamics-Based Interactive Learning Environments. Simulation \& Gaming, 31(2), 178.

9. Resnick, M., Berg, R., \& Eisenberg, M. (2000). Beyond Black Boxes: Bringing Transparency and Aesthetics Back to Scientific Investigation. The Journal of the Learning Sciences, 9(1), 7--30.

10. Roth, W. (1993). An investigation of problem framing and solving in a grade 8 open-inquiry science program. The Journal of the Learning Sciences, 3(2), 165-204.

11. Williamson, V. \& Abraham, M. (1995). The effects of computer animation on the particulate mental models of college chemistry students. Journal of Research in Science Teaching, 32, 521-534

12. Njoo, M., \& de Jong, T. (1993). Exploratory Learning with a Computer Simulation for Control Theory: Learning Processes and Instructional Support. Journal of Research in Science Teaching, 30(8), 821--844.

13. Winn, W. (2002). Research into Practice: Current Trends in Educational Technology Research: The Study of Learning Environments. Educational Psychology Review, 14(3), 331--351.

14. Bodemer, D., Ploetzner, R., Bruchmuller, K., \& Hacker, S. (2005). Supporting learning with interactive multimedia through active integration of representations. Instructional Science, 33(1), 73--95.

15. Patton, M.Q. (2002). Qualitative Research \& Evaluation Methods (3rd ed). Sague Publications.

16. Black, P. \& Wiliam, D (1998) Assessment and Classroom Learning. Assessment in Education, 5(1), 7-71.

17. Wiggins, G., \& McTighe, J. (1997). Understanding by Design: Alexandria, VA: Association for Supervision and Curriculum Development. 